\title{
Thermodynamique des systèmes micellaires \\ Partie 1 : Volumes molaires apparent et partiel des alcanoates de sodium selon le modèle d'action de masse (MAM)
}

\author{
S. M. Hajii* et M. W. O. Sellahi \\ Laboratoire de Chimie Organique et d'Etudes Physico-Chimiques (LCOEPC), E.N.S. \\ Takaddoum, Avenue Oued Akreuch, B.P. 5118, Rabat, Maroc
}

(Reçu le 05 Mai 2005, accepté le 10 Septembre 2005)

*Correspondance et tirés à part, e-mail : hajii_ms@yahoo.com

\section{Résumé}

Des mesures de densités ont permis d'obtenir les masses volumiques des solutions aqueuses de la série homologue des alcanoates de sodium du formiate $(n=0)$ à l'undécanoate $(\mathrm{n}=10)$. Les résultats ont été analysés selon le modèle d'action de masse (MAM). Ceci a permis de déterminer avec précision les différents domaines de la formation de la micelle pour chacun des homologues. Ainsi la $1^{\text {ère }}$ et parfois la $2^{\text {ème }}$ concentration micellaire critique ont été déterminées. Cette méthode d'analyse a permis de calculer les volumes molaires apparent et partiel de chacun des homologues, les volumes molaires apparents d'excès ainsi que la variation du volume durant la micellisation. Les interactions soluté-soluté et soluté-solvant ont été discutées.

Mots-clés : Densimétrie, volumes molaires apparent et partiel, volume d'excès, micelles, interactions soluté-solvant et soluté-soluté, alcanoates de sodium.

\section{Abstract}

\section{Thermodynamics of micellar systems}

Part I : Apparent and partial molar volumes of sodium alkanoates according to the mass-action model (MAM)

Densities of the homologous sodium caboxylates were measured with a flow densimeter. Results were analysed according to the mass-action model. The first and some times the second micellar critical concentrations were determined. The apparent and partial molar volumes were calculated as well as the excess volumes. The solute-solute and solutesolvent interactions were discussed.

Keywords : Densimetry, partial and apparent molar volumes, micelles, excess volumes, solute-solvent and solute-solute interactions, sodium alcanoates. 


\section{Introduction}

La connaissance des propriétés volumiques est utile à la compréhension des diverses interactions moléculaires [1]. Différents phénomènes d'associations $[2,3]$ et d'agrégations de type micellaire dans les solutions aqueuses des composés ioniques [4], d'alcools aliphatiques $[2,5]$ et d'alcane diols et triols $[6,7]$ ont été mis en évidence.

La série homologue des alcanoates de sodium a fait l'objet de nombreuses études par différentes méthodes. Ces études demeurent très fractionnées et n'ont jamais englobé un grand nombre d'homologues.

Ainsi Umémura [8], par spectroscopie infrarouge sur des solutions deutérées de cette série, du propionate au n-décanoate de sodium, a dégagé les premières concentrations micellaires critiques $\left(\mathrm{cmc}_{1}\right)$. King [9] a montré par une méthode de tassement des densités que les changements des volumes de ces sels en solution aqueuse sont liés à l'ionisation de leurs acides et ceci pour le formiate, l'acétate et le n-butyrate de sodium. Henriksson [10] a pu déterminer la $\mathrm{cmc}$ du n-hexanoate de sodium par relaxation spinréseau $d u{ }^{13} \mathrm{C}$. Alors que Lindenbaum [11] a exploité les données des solutions très concentrées de l'acétate, butyrate, valérate, heptanoate et pélargonate de sodium et a calculé les quantités d'excès : $\Delta \mathrm{G}^{\mathrm{Ex}}, \Delta \mathrm{H}^{\mathrm{Ex}}$ et $\Delta \mathrm{S}^{\mathrm{Ex}}$.

Par ailleurs Sakurai [12] a calculé les volumes molaires apparents à dilution infinie pour la série des alcanoates de sodium du formiate au pélargonate. Dans une étude, Leduc [13] a déterminé les volumes molaires apparents ainsi que les capacités calorifiques des solutions aqueuses à dilution infinie de l'acétate et de l'octanoate de sodium puis il a dégagé la cmc de ce dernier. Watanabe [14] a montré que la micelle de l'octanoate de sodium en milieu aqueux est très stable avec la participation dynamique de 15 monomères. Douheret [2] a déterminé, dans un large domaine de concentration, les masses volumiques des solutions aqueuses des alcanoates de sodium de l'acétate à l'octanoate. Par ailleurs, une étude microcalorimétrique effectuée sur la série des alcanoates de sodium du formiate à l'undécanoate [6] a montré l'existence des $\mathrm{cmc}(\mathrm{s})$ et a permis la mise en évidence, lors du passage de la solution du milieu dispersé au milieu

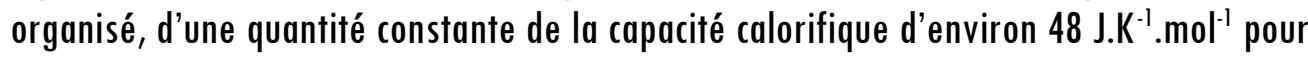
les composés formant des micelles vraies.

Le but de ce travail est d'étudier les solutions aqueuses de la série homologue des alcanoates de sodium $\mathrm{H}\left(\mathrm{CH}_{2}\right)_{n} \mathrm{COO}, \mathrm{Na}$ avec $\mathrm{n}=0$ à 10 selon le modèle d'action de masse (MAM). Nous calculons ensuite les volumes molaires apparent et partiel puis les volumes d'excès et enfin nous discutons les interactions croissantes des liaisons hydrophobes qui se manifestent à fur et à mesure de l'allongement de la chaîne hydrocarbonée. 
Dans un prochain article, nous étudierons la même série homologue mais selon la méthode de la pseudo-phase (MPP). Nous verrons comment les deux méthodes aboutissent aux mêmes résultats et à la même conclusion.

\section{Matériel et méthodes}

\section{2-1. Rappels théoriques}

De nombreux auteurs ont utilisé, pour le traitement des données expérimentales, soit le modèle d'action de masse $[15,16]$ soit le modèle de la peudo-phase $[2,17]$. Nous donnons ici un bref rappel du modèle d'action de masse.

Le modèle d'action de masse (MAM): considère que l'équilibre entre les micelles et les monomères peut expliquer l'existence d'une concentration critique assez stricte dite concentration micellaire critique (cmc), au-dessus de laquelle commence la formation des micelles [18]. Lors de la formation des micelles les grandeurs thermodynamiques tels les volumes molaires, les capacités calorifiques, l'entropie, le coefficient osmotique, etc., subissent des modifications notables. Ainsi par exemple, dans la structure des micelles, l'entropie diminue puisque les chaînes hydrophobes auront moins de liberté que dans le monomère du fait que les queves paraffiniques dans l'eau sont contraintes à présenter le minimum de contact possible huile/eau. Dans le calcul de l'enthalpie libre, Debye [20] a considéré que lors de la formation de la micelle, cette quantité standard doit être minimum et donc la constante de la loi d'action de masse doit être maximum.

Ce même résonnement a été repris plus tard par Stigter et Overbeek [21]. Ils expliquent que le maximum recherché est celui de la fraction molaire de la micelle qui dénote la dimension la plus fréquente de l'espèce et non le maximum de la constante d'équilibre.

\section{2-2. Produits et techniques expérimentales}

\section{2-2-1. Produits}

Les alcanoates de sodium utilisés ont été obtenus par neutralisation de l'acide correspondant. Ces acides d'origines différentes ont été distillés sous pression réduite immédiatement avant neutralisation par une solution titrée normale de soude. Les sels obtenus, après évaporation de l'eau au bain-marie, ont été séchés à l'étuve à $110^{\circ} \mathrm{C}$ pendant 48 heures, puis au dessiccateur (sur anhydride phosphorique) pendant trois jours jusqu'à élimination totale de l'eau.

Le formiate et l'acétate de sodium (ultra pur Normaton) dont la teneur maximale en impureté est de $10^{-4} \%$, furent utilisés sans purification supplémentaire. Le propionate 
de sodium pur à $99 \%$ a été filtré à chaud, recristallisé puis lavé à l'éthanol absolu à froid. Ces trois derniers sels ont été séchés à l'étuve puis au dessiccateur comme vu précédemment.

\section{2-2-2. Techniques expérimentales}

Les densités des solutions sont déterminées à 298,15 K par un densimètre commercial version SODEV (02D) [22]. Son principe est basé sur la propriété d'un oscillateur mécanique constitué d'un tuyau en inox dans lequel circule un liquide dont on veut mesurer la masse volumique $(\rho)$. La mesure de la période de résonance $(\tau)$ de ce tuyau permet de calculer la masse volumique du fluide selon la relation suivante :

$$
p=A+K(z)^{2}
$$

$A$ et $K$ sont des constantes de calibrage du système à une température donnée.

La période correspondant à la fréquence de résonance du tuyau oscillateur est de l'ordre de $3.10^{-3} \mathrm{sec}$. Les fluctuations de la température doivent être maintenues en deçà de $10^{-3}$ ${ }^{\circ} \mathrm{C}$, car la masse volumique de la plus part des liquides varie de quelques centaines de ppm par degré C. Pour des mesures différentielles, la relation (I) se réduit à :

$$
\rho^{t^{o}}=\rho o^{t^{o}}+K t^{o}\left(\tau^{2}-\tau o^{2}\right)
$$

où $\rho_{0}$ et $\tau_{0}$ se référent au liquide de référence (généralement le solvant pur) et $t^{\circ}$ la température à laquelle on travaille.

La constante $K$ est déterminée par calibrage avec des liquides et des gaz dont les masses volumiques sont connues. Dans notre cas, nous avons utilisé l'eau bidistillée et filtrée sur des filtres millipores de $0.45 \mu \mathrm{m}\left(\rho_{0}\right.$ à $\left.25^{\circ} \mathrm{C}=0.997047 \mathrm{~g} \cdot \mathrm{cm}^{-3}[23,24]\right)$ et l'azote $\mathrm{N} 48$ dont la pureté est supérieure à $99.998 \%\left(\rho_{0}\right.$ à $\left.25^{\circ} \mathrm{C}=1.14583 \times 10^{-3} \mathrm{~g} \cdot \mathrm{L}^{-1}[23,24]\right)$. $0 \mathrm{n}$ trouve $K=7.131 \times 10^{-7} \pm 10^{-10}$. Avec cette technique, les différences sur les masses volumiques peuvent être mesurées à $\pm 3 \times 10^{-6} \mathrm{~g} \cdot \mathrm{cm}^{-3}$, avec environ $5 \mathrm{~cm}^{3} \mathrm{du}$ liquide et en $5 \mathrm{mn}$ de temps. Cette incertitude correspond approximativement à $\pm 10^{-4}$ mole. $\mathrm{kg}^{-1}$ sur la concentration des électrolytes simples dans l'eau.

Cette technique est très sensible aux modifications de structure qui interviennent dans la solution. Elle a l'avantage de ne pas modifier l'équilibre des espèces en solution par l'introduction de substances nouvelles. A partir des masses volumiques, on calcule les différentes grandeurs molaires tels que le volume molaire apparent $\Phi_{\mathrm{v}}$ et le volume molaire partiel $\overline{V_{2}}$ via les relations suivantes:

$$
\overline{\mathrm{V}}_{2}=\left[\frac{\delta V}{\delta n_{2}}\right]_{\mathrm{T}}, \mathrm{P}, n_{2}
$$

puisque d'une façon générale :

\section{S. M. Hajii et al.}




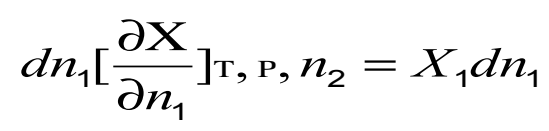

avec $\mathrm{X}=n_{1} \mathrm{X}_{1}+n_{2} \mathrm{X}_{2}$

ici $\quad V=n_{1} \bar{V}_{1}+n_{2} \bar{V}_{2}$

Le volume molaire apparent $\Phi_{\mathrm{v}}$ du composé dissout sera défini par :

$$
V=n_{1} V o_{1}+n_{2} \Phi_{V}
$$

$\bar{V}_{1}$ : volume molaire partiel du solvant dans la solution,

$\bar{V}_{0_{1}}$ : volume molaire du solvant pur,

$n_{l}$ : nombre de moles du solvant, $n_{2}$ : nombre de moles du soluté, d'où l'on tire :

$$
\bar{V}_{2}=\left[\frac{\partial V}{\partial n_{2}}\right]=\Phi+n_{2}\left[\frac{\partial \Phi_{V}}{\partial n_{2}}\right]
$$

Par ailleurs, si on utilise $1000 \mathrm{~g}$ d'eau, on aura $\mathrm{n}_{2}=\mathrm{m}$ et $\Phi_{\mathrm{v}}$ devient :

$$
\begin{aligned}
& \Phi_{V}=\frac{M}{\boldsymbol{\rho}}-1000\left(\frac{\boldsymbol{\rho}-\boldsymbol{\rho} \mathrm{o}}{\boldsymbol{\rho} \cdot \boldsymbol{\rho} \mathrm{O} \cdot \boldsymbol{m}}\right) \\
& \text { et } \quad \bar{V}_{2}=\Phi+m \cdot\left[\frac{\partial \Phi_{V}}{\partial m}\right]
\end{aligned}
$$

où $M$ désigne la masse molaire du corps dissous, $m$ la molalité de la solution, $\rho$ sa masse volumique et $\rho_{0}$ la masse volumique du solvant pur.

$V_{2}$ est déduit directement de $\Phi_{\mathrm{v}}[6,17,23]$. Son extrapolation à dilution infinie ainsi que celle de $\Phi v$ permet d'accéder au volume molaire apparent à dilution infinie $\overline{V_{2}^{0}}=\Phi_{V}^{0}$.

Pour les produits ioniques, selon la relation de Redlich-Meyer [25] donnée pour les électrolytes $1: 1$, on a dans la région prémicellaire :

$$
\Phi_{V}-\mathrm{A}_{V}\left(\rho_{0 . m}\right)^{\frac{1}{2}}=\Phi_{V}^{0}+\mathrm{B}_{V} m
$$

où $A_{v}$ est la pente limite de la loi Debye-Huckel ( à $25^{\circ} \mathrm{C}, A_{\nu}$ est égale à $1.686 \mathrm{~cm}^{3 / 2} \cdot \mathrm{mol}^{-3 / 2}$ [26], $1.865 \mathrm{~cm}^{3 / 2} \cdot \mathrm{mol}^{-3 / 2}[27]$ ) et $B_{v}$ un paramètre ajustable qui mesure la déviation à cette loi.

On calcule ensuite le volume molaire d'excès à partir de la relation donnée par Leduc [28] :

$$
\Phi_{V}^{\mathrm{E}_{\mathrm{X}}}=\Phi_{V}-\Phi_{V}^{0}-\mathrm{A}_{V}(C)^{\frac{1}{2}}
$$

\section{S. M. Hajii et al.}


Les solutions sont préparées par pesée avec une balance sensible à $5 \cdot 10^{-5} \mathrm{~g}$. L'eau utilisée est déminéralisée, bidistillée puis filtrée sur des filtres millipore de $0.45 \mu \mathrm{m}$.

\section{Résultats et discussion}

\section{3-1. Modèle d'action de masse (M.A.M.)}

Ce modèle est retenu par les auteurs $[29,30]$ comme le modèle le plus fondamental car il considère que l'équilibre entre les micelles et les monomères peut expliquer l'existence d'une concentration micellaire critique (cmc) assez stricte, au-dessus de laquelle commence la formation des micelles $[18,31]$.

\section{3-1-1. L'effet de la longueur de la chaîne alkyle sur cmc}

Les courbes $\Phi v=f\left(V_{m}\right)$ et $V_{2}=f\left(V_{m_{\text {moy }}}\right)$ sont représentées par les Figures 1 et 2. Elles ont une allure en forme de " $S$ " étalé, notamment pour les produits ayant des chaînes hydrocarbonées moyennes et longues $(n=6$ à 10). Ces produits présentent des interactions hydrophobes avec parfois la formation des micelles.

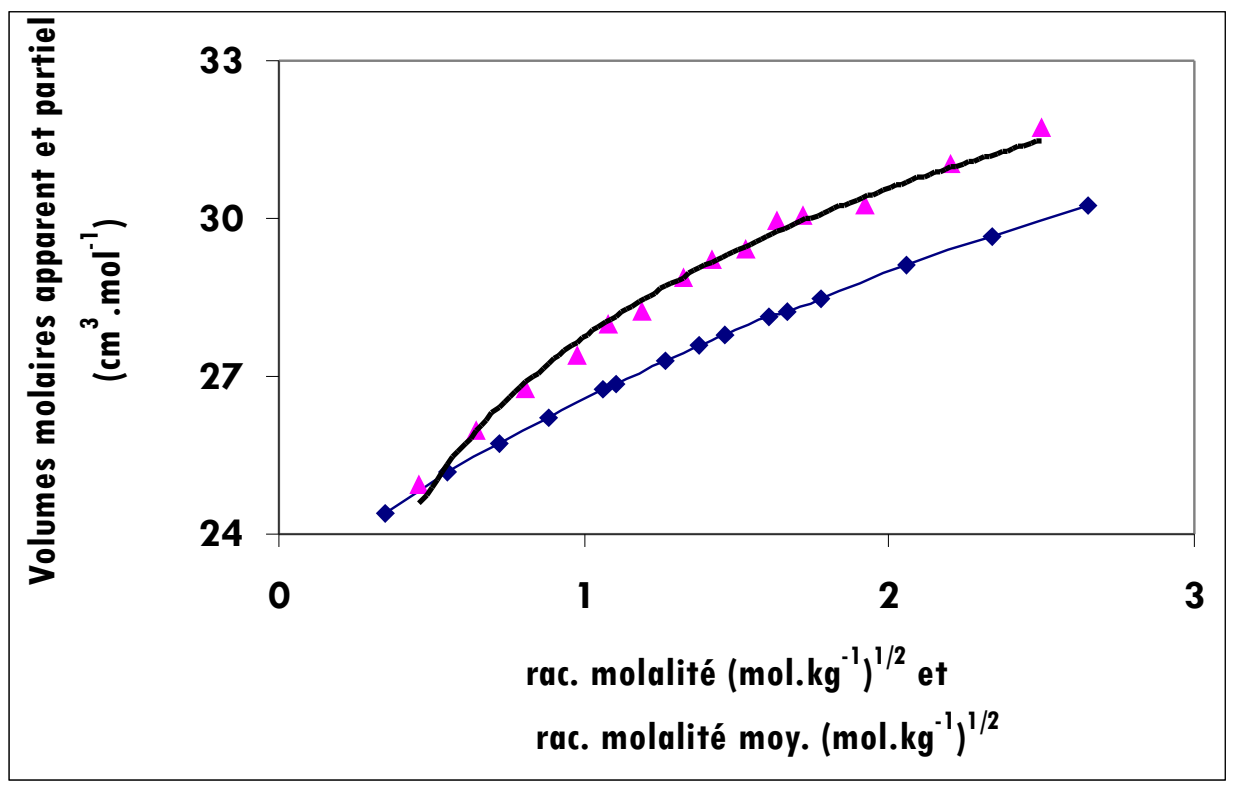

Figure 1 : Volumes molaires apparent $(\bullet)$ et partiel $(\mathbf{\Delta})$ du formiate de sodium $(n=0)$ selon le modèle d'action de masse (MAM) 


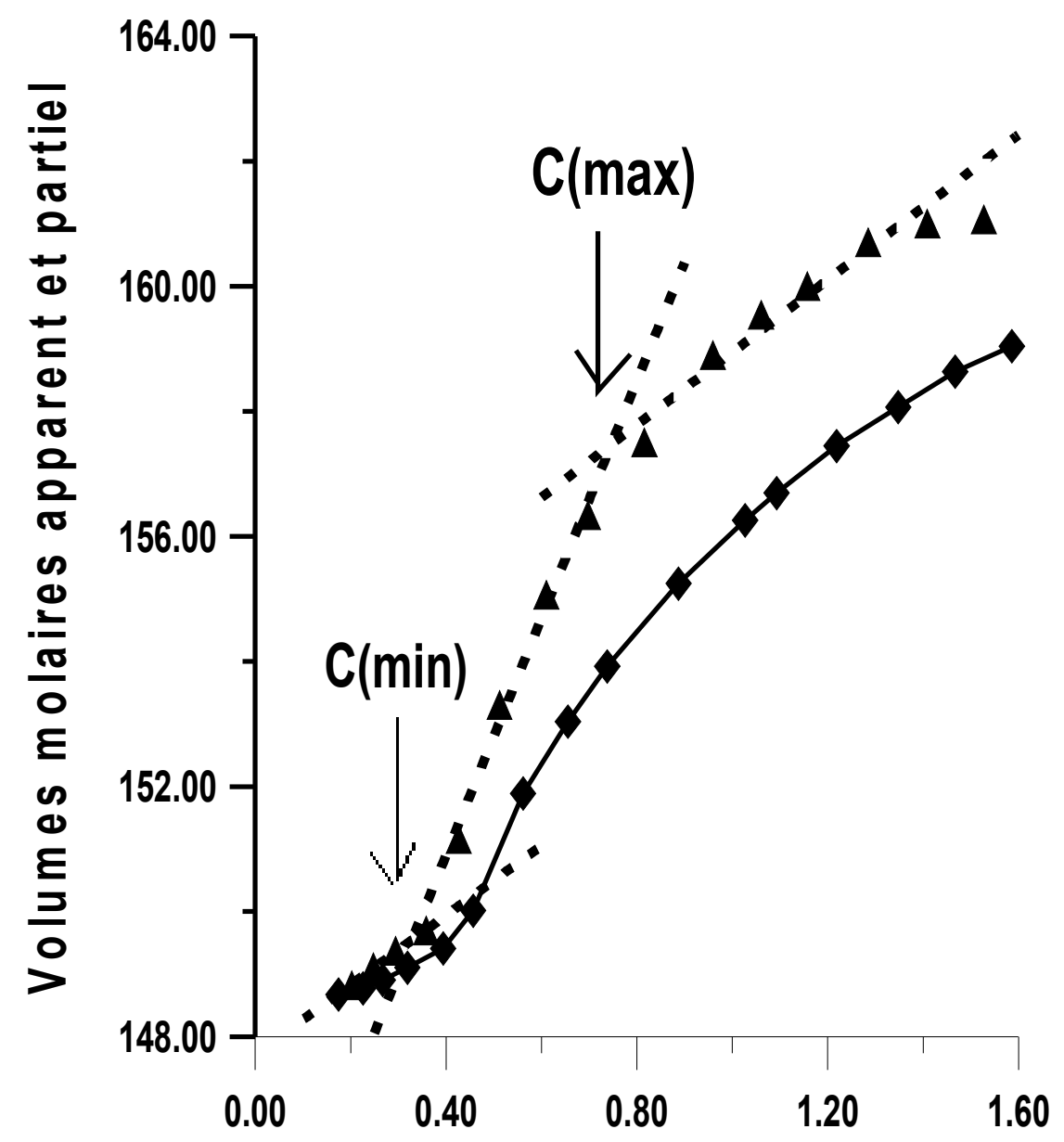

Figure 2 : Volumes molaires apparent $(\bullet)$ en $\mathrm{cm}^{3} \cdot \mathrm{mol}^{l}$ et partiel(A) en $\mathrm{cm}^{3}$. $\mathrm{mol}^{l}$ du nonanoate de sodium $(n=8)$ selon le modèle d'action de masse (MAM)

La concentration micellaire critique (cmc) est prise comme concentration à partir de laquelle se manifeste une brusque variation du volume molaire apparent. Les différentes valeurs des cmc(s) sont consignées dans le Tableav 1 . La diversité des résultats rencontrés dans la littérature concernant les $\mathrm{cmc}(\mathrm{s})$ tient naturellement de la diversité des techniques et des méthodes utilisées. 
Tableau 1: Concentrations micellaires critiques des alcanoates de sodium $a ̀ 25^{\circ} \mathrm{C}$

\begin{tabular}{|c|c|c|c|}
\hline $\mathrm{n}$ & $\begin{array}{c}\mathrm{cmc}_{1} \\
\left(\mathrm{~mol}^{\left.-\mathrm{L}^{-1}\right)}\right)\end{array}$ & $\mathrm{cmc}_{1}$ (par d'autres méthodes) $\left(\mathrm{mol}^{\left.\mathrm{L} . \mathrm{L}^{-1}\right)}\right.$ & $\begin{array}{c}\mathrm{cmc}_{2} \\
\left(\mathrm{~mol}^{\left.-\mathrm{L}^{-1}\right)}\right.\end{array}$ \\
\hline 0 & - & - & - \\
\hline 1 & - & - & - \\
\hline 2 & - & - & - \\
\hline 3 & 2,19 & $3,99^{\mathrm{m}} ; 2,65^{\mathrm{a}} ; 4,45^{\mathrm{c}}$ & - \\
\hline 4 & 1,56 & $2,15^{\mathrm{m}} ; 1,75^{\mathrm{a}} ; 1,80^{\mathrm{f}} ; 2,90^{\mathrm{d}}$ & - \\
\hline 5 & 1,032 & $1,46^{\mathrm{m}} ; 1,15^{\mathrm{a}}$ & - \\
\hline 6 & 0,63 & $0,81^{\mathrm{m}} ; 0,70^{\mathrm{a}} ; 0,55^{\mathrm{f}} ; 0,79^{\mathrm{c}, 9} ; 1,06^{\mathrm{h}}$ & - \\
\hline 7 & 0,37 & $0,409^{\mathrm{m}} ; 0,40^{\mathrm{a}, \mathrm{ch}} ; 0,32^{\mathrm{f}} ; 0,41^{\mathrm{b}} ; 0,45^{\mathrm{k}} ; 0,405^{\mathrm{g}} ; 0,351^{\mathrm{l}} ; 0,36^{\mathrm{e}}$ & 0,1347 \\
\hline 8 & 0,16 & $0,23^{m} ; 0,17^{\dagger} ; 0,21^{g}$ & 0,917 \\
\hline 9 & 0,10 & $0,13^{\mathrm{m}} ; 0,09^{\mathrm{f}} ; 0,109^{\mathrm{g}} ; 0,10^{\mathrm{i}}$ & 0,89 \\
\hline 10 & 0,05 & $0,075^{\mathrm{m}}$ & 0,343 \\
\hline
\end{tabular}

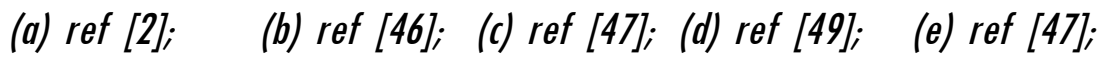

(f) ref $[49,50] ;$ (g) ref $[4] ; \quad$ (h) ref [50]; (i) ref [48]; (i) ref [8];

(k) ref $[22] ; \quad$ (I) ref $[51] ; \quad(m)$ ref $[6]$

La variation de la cmc en fonction de $\mathrm{n}$ (la longueur de la chaîne hydrocarbonée) (Figure 3 ), obéit à la relation de Klevens [32] :

$$
\log (\mathrm{cmc})=A+B \cdot n
$$

On constate que deux lois distinctes sont nécessaires pour rendre compte de l'allure de la courbe. Une première droite pour les $\mathrm{cmc}(\mathrm{s})$ des produits formant des micelles $(\mathrm{n}=6$ à 10) puis une deuxième pour les produits ayant $n=2$ à 6 . On pourrait penser qu'il s'agit, pour ces derniers, uniquement d'associations moléculaires à très peu de monomères plutôt qu'à une association micellaire.

Des résultats analogues ont été observés [2,33] pour la même série des alcanoates de sodium de $\mathrm{n}=2$ à 9 . 


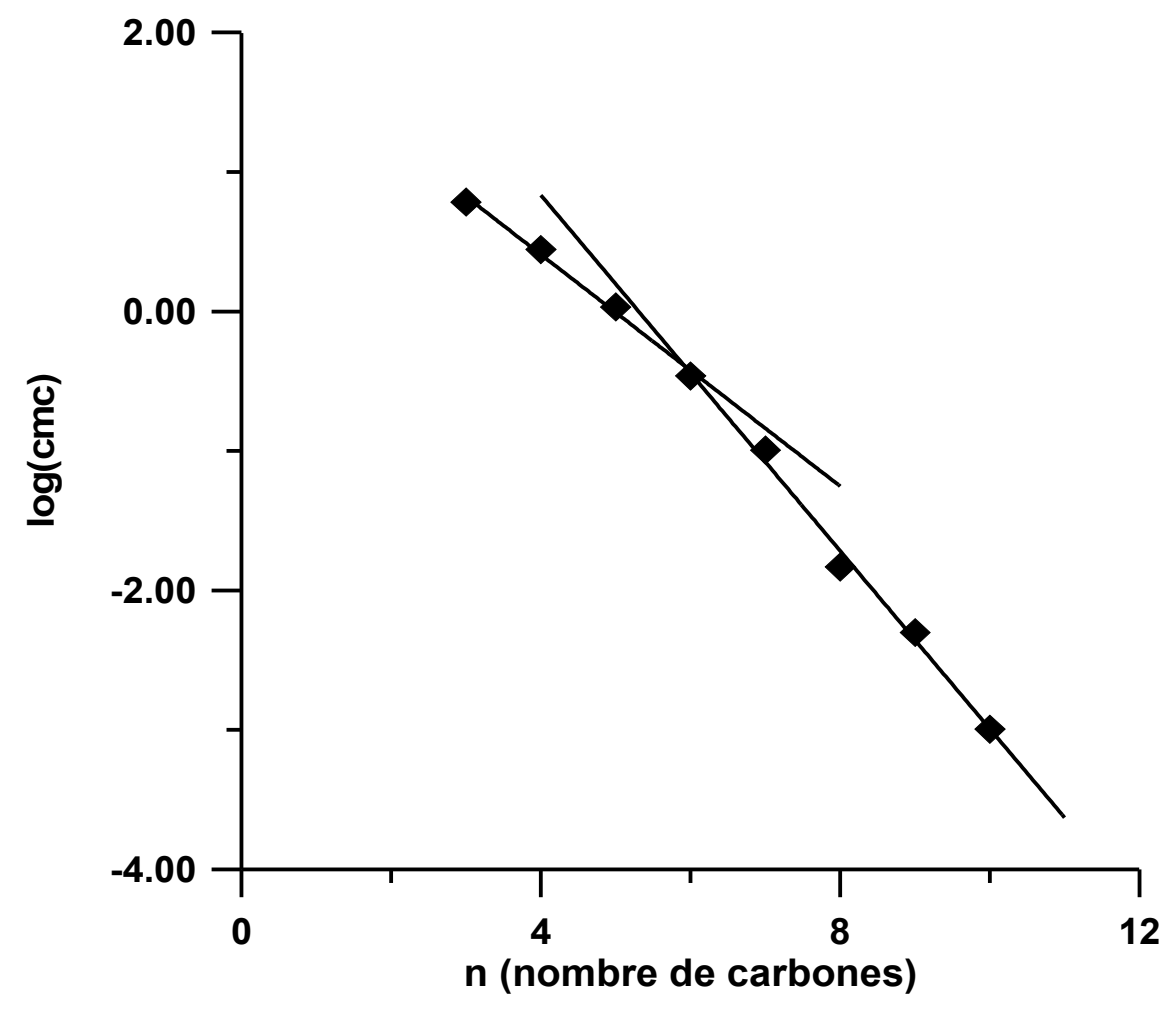

Figure 3 : Variation du log de la cmc en fonction du nombre d'atomes de carbone (n) dans la chaîne aliphatique

L'application d'un programme de régression linéaire sur les six derniers points permet de déterminer $A=3,38 \pm 0,01$ et $B=-0,64 \pm 0,01$.

$$
B=-\Delta G / 2,303 K T(I+K g)
$$

$\Delta G$ est la variation d'énergie libre de Gibbs correspondant au transfert d'un - $\mathrm{CH}_{2}$ de la phase aqueuse à la phase micellaire. $\mathrm{Kg}$ est un coefficient caractérisant l'effet des ions antagonistes $\left(\mathrm{Na}^{+}, \mathrm{K}^{+}, \mathrm{Cl}, \mathrm{Br}\right.$ etc. ). Il est toujours compris entre 0,4 et 0,6 pour un anion ou un cation et il est nul pour un non ionique.

En prenant $\mathrm{Kg}=0,56$ comme l'a suggéré Coudert [35], on trouve $\Delta G=-2,45 \mathrm{~kJ}^{\mathrm{mol}}{ }^{-1}$. Cette valeur s'accorde bien avec les résultats trouvés par d'autres auteurs : $-2,89$ $\mathrm{kJ} \mathrm{mol}{ }^{-1}$ pour une série homologue d'alcanetriols-1,2,3 [35] et $-0,69 \mathrm{~kJ}^{\mathrm{mol}} \mathrm{l}^{-1}$ pour une série d'octaétylène-n-alkyl éthers [36]. 


\section{3-1-2. Variation des volumes molaires apparent et partiel}

Les courbes représentatives des volumes molaires partiels en fonction de la racine carrée de la molalité moyenne.

$\bar{V}_{2}=f(\bar{m})^{\frac{1}{2}}$ sont données dans les Figures 1 et 2 . Pour $5 \leq \mathrm{n} \leq 10$, ces courbes présentent trois portions de droites qui se coupent en $C_{\min }$ et $C_{\max }$ correspondant aux volumes molaires partiels et $\bar{V}_{2(\min )} \bar{V}_{2(\max )}$ respectivement avant et après la micellisation. La zone de micellisation est délimitée par $C_{\min }$ et $C_{\max }$.

A dilution infinie les courbes $\Phi_{V}=f(\bar{m})^{\frac{1}{2}}$

$\bar{V}_{2}=f(\bar{m})^{\frac{1}{2}}$ vont s'extrapoler à $\Phi_{V}^{0}=\bar{V}_{2}^{0}$

L'application de l'équation (1I)à la zone de concentration très en dessous de la cmc se traduit par une droite pour tous les composés étudiés, ce qui permet d'accéder à $\Phi_{\mathrm{v}}^{0}$ et $B_{v}$ (Tableau 2).

Les valeurs trouvées de $\Phi^{0}{ }_{\mathrm{v}}$ pour différents produits de la série des alcanoates sont en bon accord avec celles de $n=0,1,2$ [9], de $n=2,3,4$ [2] et de $n=7$ [13]. Cependant elles demeurent légèrement basses pour $\mathrm{n}=5$ et 6 par rapport à celles de Sakurai [12] et Douheret [2]. Tandis que $\Phi^{0}$ de $n=9$ est légèrement supérieur à celles de King [2] et de Kale [19].

Les valeurs du paramètre $\boldsymbol{B}_{v}$, caractérisant les interactions soluté-soluté, sont environ zéro pour $\mathrm{n}=1$ à 4 , puis augmentent avec l'allongement de la chaîne hydrophobe. Toutes ces valeurs demeurent légèrement supérieures à celles de Sakurai $[12,37]$ à l'exception de celles pour $\mathrm{n}=1$ et 2 qui restent concordantes. Par étude microcalorimétrique de cette série des alcanoates de sodium on a déterminé [6] des valeurs de $B_{c}$ (les capacités molaires d'excès) toutes positives et croissantes. Par ailleurs, les bromures de $\mathrm{R}(-)\left(\mathrm{N}, \mathrm{N}-\right.$ diméthyl, $\mathrm{N}-\mathrm{alkyl}\left(\mathrm{C}_{\mathrm{n}} \mathrm{H}_{2 \mathrm{n}+1}\right)$ ammonio)-2 butanol [6], avec $\mathrm{n}=10$, 11 et 12, produits formant de vraies micelles en solution aqueuse, ont dégagé des valeurs de $B_{v}$ d'autant plus négatives que la chaîne hydrophobe est longue.

Une étude de ce paramètre en fonction de la température est nécessaire pour pouvoir approfondir la discussion ef tirer une quelconque conclusion. La variation du volume durant la micellisation correspond à :

$$
\Delta V_{m}=\bar{V}_{2(\max )}-\bar{V}_{2(\min )}
$$


Tableau 2 : Grandeurs volumiques calculées selon le modèle d'action de masse

\begin{tabular}{|c|c|c|c|c|c|c|c|c|c|}
\hline $\mathrm{n}$ & $\begin{array}{l}\bar{V}_{2(\mathrm{~min})} \\
\mathrm{cm}^{3} \cdot \mathrm{mol}^{-1}\end{array}$ & $\begin{array}{l}\bar{V}_{2(\max )} \\
\mathrm{cm}^{3} \cdot \mathrm{mol}^{-1}\end{array}$ & $\begin{array}{c}\mathrm{B}_{\mathrm{v}} \\
\mathrm{cm}^{3} \cdot \mathrm{mol}^{-1} \cdot \mathrm{Kg}\end{array}$ & $\begin{array}{c}\Phi_{V}^{0}=\bar{V}_{2}^{0} \\
\mathrm{~cm}^{3} \cdot \mathrm{mol}^{-1}\end{array}$ & $\underset{\mathrm{cm}^{3} \cdot \mathrm{mol}^{-1}}{\Delta \bar{V}_{2}^{0}}$ & $\begin{array}{c}\Delta \bar{V}_{m} \\
\mathrm{~cm}^{3} \cdot \mathrm{mol}^{-1}\end{array}$ & $\begin{array}{c}\mathrm{C}_{\min } \\
\mathrm{mol} . \mathrm{L}^{-1}\end{array}$ & $\begin{array}{c}\mathrm{C}_{\max } \\
\mathrm{mol} . \mathrm{L}^{-1}\end{array}$ & $\underline{C}_{\max }$ \\
\hline 0 & - & - & $\begin{array}{c}0,12 \\
0,0^{a}\end{array}$ & $\begin{array}{c}25,03,25,15^{a} \\
25,056^{c}\end{array}$ & - & - & - & - & - \\
\hline 1 & - & - & $\begin{array}{l}0,01 \\
0,0^{a}\end{array}$ & $\begin{array}{c}39,28,39,39^{b} \\
39,22^{c}, 39,18^{d} \\
39,24^{i}\end{array}$ & 14,25 & - & - & - & - \\
\hline 2 & - & - & $\begin{array}{c}-0,01 \\
0,0^{\circ}\end{array}$ & $\begin{array}{c}53,86 \\
53,71^{\mathrm{a}}, 53,8^{\mathrm{h}}\end{array}$ & 14,58 & - & - & - & - \\
\hline 3 & - & - & $\begin{array}{l}0,12 \\
-0,2^{a}\end{array}$ & $\begin{array}{c}69,02, \\
69,32^{\mathrm{a}}, 69,188^{\mathrm{c}}, \\
69,35^{\mathrm{h}}\end{array}$ & 15,31 & - & - & - & - \\
\hline 4 & - & - & $\begin{array}{l}-0,09 \\
-0,21^{b}\end{array}$ & $\begin{array}{c}85,04,85,69^{b} \\
85,2^{\mathrm{h}}\end{array}$ & 16,02 & - & - & - & - \\
\hline 5 & 103,14 & 111,55 & $\begin{array}{c}0,25 \\
-0,77^{b}\end{array}$ & $\begin{array}{c}100,69,101,36^{b}, \\
101,35^{\mathrm{h}}\end{array}$ & 15,65 & 3,21 & 0,94 & 1,96 & 2,08 \\
\hline 6 & 119,99 & 125,95 & $\begin{array}{l}-0,14 \\
-0,57^{b}\end{array}$ & $\begin{array}{c}116,86,117,05^{b}, \\
117,4^{\mathrm{h}}\end{array}$ & 16,17 & 11,11 & 0,30 & 0,82 & 2,70 \\
\hline 7 & 133,11 & 142,64 & $\begin{array}{l}0,54 \\
-0,46^{b}\end{array}$ & $\begin{array}{c}132,02,133^{b, c}, \\
132,12^{d}\end{array}$ & 15,16 & 8,83 & 0,25 & 0,44 & 1,76 \\
\hline 8 & 149,02 & 158,01 & $\begin{array}{l}1,52 \\
-0,14^{b}\end{array}$ & $148,29,148,73^{b}$ & 16,27 & 11,14 & 0,12 & 0,24 & 2,0 \\
\hline 9 & 165,51 & 172,03 & 3,25 & $\begin{array}{c}164,63,163,93^{c} \\
164,17^{f}\end{array}$ & 16,34 & 8,87 & 0,095 & 0,19 & 1,89 \\
\hline 10 & 181,01 & 187,10 & 9,80 & 179,95 & 15,3 & 9,77 & 0,047 & 0,078 & 1,65 \\
\hline
\end{tabular}

(a) $\operatorname{ref}[45]$, (b) $\operatorname{ref}[12]$, (c) ref [9], (d) ref [13], (e) ref [25], (f) ref [19], (h) ref [2], (i) ref [43], (i) ref [44].

Les résultats obtenus pour $\Delta \bar{V}_{m}$ sont reportés dans le tableau 2. Seule la série à $\mathrm{n} \geq 5$ est exploitable. Nous remarquons que toutes ces valeurs sont supérieures à $5 \mathrm{~cm}^{3} \cdot \mathrm{mol}^{-1}$ à l'exception de celle de l'hexanoate de sodium pour lequel $\Delta \bar{V}_{m}=3,21 \mathrm{~cm}^{3} \cdot \mathrm{mol}^{-1}$. Cela montre un comportement micellaire pour les produits ayant une chaîne hydrocarbonée supérieure ou égale à six carbones $(\mathrm{n} \geq 6)$, car l'association des portions hydrophobes aux voisinages des cmc(s) entraine la formation de vraies micelles tout en diminuant le contact avec l'eau. D'où une augmentation des volumes molaires apparent et partiel $[38,39]$. 
Ces valeurs de $\Delta \bar{V}_{m}$ sont du même ordre de grandeur que celles trouvées pour les bromures de $\mathrm{R}(-)$ (N,N-diméthyl,N-alkyl $\left(\mathrm{C}_{n} \mathrm{H}_{2 \mathrm{n}+1}\right)$ ammonio)-2butanol [6], avec $\mathrm{n}=10,11$ et 12. Dans une étude comparative [40], les Auteurs ont trouvé $\Delta \bar{V}_{m}=5 \mathrm{~cm}^{3} \cdot \mathrm{mol}^{-1}$ et $5,76 \mathrm{~cm}^{3} \cdot \mathrm{mol}^{-1}$ respectivement pour l'hexanediol-1,2 et l'octanetriol-1,2,3.

La valeur de $\Delta \bar{V}_{m}=3,21 \mathrm{~cm}^{3} \cdot \mathrm{mol}^{-1}$ de l'hexanoate de sodium ( $\left.\mathrm{n}=5\right)$ demeure analogue à celles trouvées respectivement pour l'hepanetriol-1,2,3 et l'hexanetriol-1,2,3 $(3,73$ $\mathrm{cm}^{3} \cdot \mathrm{mol}^{-1}$ et 2,33 $\mathrm{cm}^{3} \cdot \mathrm{mol}^{-1}$ ) [35] et pour lesquels on a montré [6] par diffusion de la lumière laser, qu'ils ne possèdent pas d'interactions hydrophobes type micellaire.

L'hexanoate de sodium $(n=5)$ serait le produit charnière entre ceux qui formeraient des micelles et ceux qui n'en formeraient pas.

\section{3-1-3. Contribution volumique par incrément $-\mathrm{CH}_{2}$}

En milieu aqueux, la contribution du volume molaire partiel du groupe méthylène $\overline{V^{0}}\left(\mathrm{CH}_{2}\right)$ est donnée par la relation :

$$
\overline{V^{0}}\left(\mathrm{CH}_{2}\right)=\frac{\left[\overline{V^{0}}\left(n_{i}\right)-\bar{V}^{0}\left(n_{j}\right)\right]}{\left(n_{i}-n_{j}\right)}
$$

Où $n_{i}$ et $n_{i}$ représentent le nombre de groupes méthylènes du produit considéré.

Ainsi on remarque que la valeur

moyenne de $\overline{V^{0}}\left(\mathrm{CH}_{2}\right)$ de l'hexanoate, heptanoate, octanoate, nonanoate, décanoate et undécanoate de sodium, est de l'ordre de $15,9 \mathrm{~cm}^{3} \cdot \mathrm{mol}^{-1}$, ce qui correspond au volume molaire partiel d'un - $\mathrm{CH}_{2}$ libéré de l'influence des groupes polaires [12].

Cette valeur est en bon accord avec la valeur $15,9 \mathrm{~cm}^{3} \cdot \mathrm{mol}^{-1}[5,12,41,42]$ trouvée dans le milieu aqueux à $25^{\circ} \mathrm{C}$.

La contribution $\overline{V^{0}}$ par groupe $\mathrm{CH}_{2}$ ne serait constante que pour les homologues à courtes chaînes. D'après Sakurai [35], cette déviation peut être expliquée par l'influence des groupes polaires sur l'hydratations hydrophobe du groupe méthylène.

En milieu hydrocarboné, les contributions par incrément $-\mathrm{CH}_{2}$ peuvent être évaluées pour une série homologue en comparant les résultats obtenus pour $\Phi_{v}$ et $\bar{V}_{2(\min )}$ à ceux de $V^{0}$ à dilution infinie. En effet, on remarque que pour l'hexanoate, heptanoate, octanoate, nonanoate, décanoate et undécanoate de sodium, la différence entre les volumes minimums du produit $\mathrm{n}$ et du produit $\mathrm{n}-1 V_{2(\min )}$ est d'environ $15,6 \mathrm{~cm}^{3} \cdot \mathrm{mol}^{-1}$.

\section{S. M. Hajii et al.}


On constate que la contribution diminue lorsqu'on passe du milieu aqueux $(15,96$ $\left.\mathrm{cm}^{3} \cdot \mathrm{mol}^{-1}\right)$ au milieu hydrocarboné $\left(15,66 \mathrm{~cm}^{3} \cdot \mathrm{mol}^{-1}\right)$, ce qui est attribué à la formation d'agrégats micellaires.

Coudert et al. [35] est arrivé aux mêmes conclusions en étudiant la série des alcanetriols-1,2,3 dans les zones pré et post micellaires.

\section{3-1-4. Variation des volumes molaires apparents d'excès}

La représentation graphique des volumes molaires d'excès $\left(\Phi_{\mathrm{v}}{ }^{\mathrm{Ex}}\right)$ de la série des alcanoates de sodium du formiate à l'undécanoate en fonction de la racine carrée de la concentration donnée selon l'équation (12), est présentée à la Figure 4.

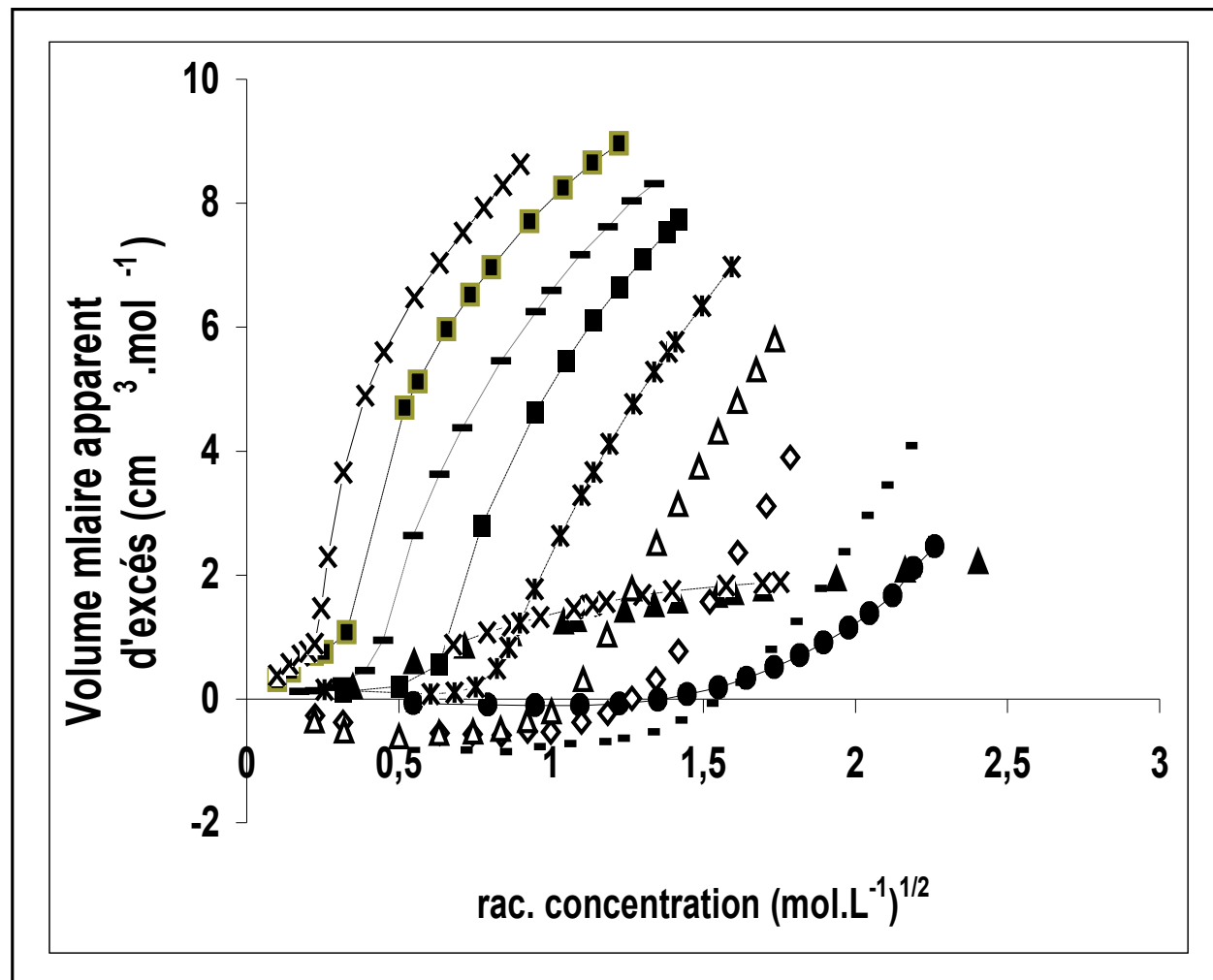

Figure 4 : Volumes molaires apparents d'excès $\left(\Phi_{v}{ }^{E x}\right)$ de la série homologue des alcanoates de sodium : $(\mathbf{A}) n=0,(-. x-) n=1,.(\bullet) n=2,(-) n=3,()) n=4$, $(\triangle) n=5,\left(^{*}\right) n=6,() n=7,(-) n=8,(-) n=9,(x) n=10$ 
On remarque que :

- Pour le formiate et l'acétate ( $n=0$ et 1 ), au fur et à mesure que la concentration crô̂t, les valeurs de $\Phi_{\mathrm{v}}{ }^{\mathrm{Ex}}$ augmentent et demeurent positives.

- Les courbes du propionate, butanoate, pentanoate et de l'hexanoate ( $n=2,3,4,5$ ) passent par des valeurs négatives de $\Phi_{\mathrm{v}}{ }^{\mathrm{Ex}}$, puis augmentent pour devenir positives vers les fortes concentrations.

- Les valeurs de $\Phi_{\mathrm{v}}^{\text {Ex }}$ de l'heptanoate, octanoate, nonanoate, décanoate et undécanoate ( $\mathrm{n}=6,7,8,9$ et 10 ) sont toutes positives et présentent un point d'inflexion plus marqué pour les quatre derniers produits.

$\Phi_{\mathrm{v}}{ }^{\mathrm{Ex}}$ représente ici le paramètre $B_{\mathrm{v}}$ de l'équation (12), qui reflète le comportement des interactions soluté-soluté autres que ceux du type Debye-Huckel à longue distance.

Leduc [13] a montré que $\Phi_{\mathrm{v}}{ }^{\mathrm{Ex}}$ de certains produits tensioactifs tel que l'octanoate de sodium augmente avec la concentration, tandis que ceux des bromures de tetrabutyl ammoniums présentent un minimum.

\section{Conclusion}

Grâce aux traitements appropriés des résultats, il a été possible de montrer les changements de structure survenus dans de larges domaines de concentrations étudiées pour chaque amphiphile. Nous avons montré l'existence de la $1^{\text {ère }}$ et parfois la $2^{\text {ème }}$ concentration micellaire critique. Les volumes molaires apparents et partiels ainsi que les volumes d'excès correspondants aux différents homologues ont été déterminés.

Ainsi l'existence des agrégations micellaires est à exclure pour le formiate et l'acétate de sodium $(\mathrm{n}<2$ ), pour lesquels seule une phase de dispersion du tensioactif en milieu aqueux est à considérer.

Le propanoate, le butanoate, le pentanoate ef l'hexanoate de sodium $(2 \leq \mathrm{n} \leq 5)$ présentent une structure prémicellaire stabilisée à concentration élevée. L'hexanoate de sodium serait le produit charnière entre ceux qui formeraient des micelles et ceux qui n'en formeraient pas. L'heptanoate, l'octanoate, le nanoate, le décanoate et l'undécanoate de sodium $(6 \leq \mathrm{n} \leq 10)$ sont les seuls produits de cette série qui présentent des micelles, au sens prédéfini plus haut, et qui forment de vraies micelles.

Par ailleurs, nous avons discuté les interactions hydrophobes croissantes qui apparaissent avec l'allongement de la chaîne conduisant à la formation des micelles surtout pour les homologues ayant une chaîne hydrocarbonée à $\mathrm{n} \geq 5$. 


\section{Remerciements}

Nous tenons à remercier Monsieur le Professeur R. R. Durand de l'Université de Paris 13 pour les fructueuses discussions concernant ce travail.

\section{Références}

[1] - F.J. Millero, Chem. Rev., 7 (1971) 147-76

[2] - G. Douheret et A. Viallard, J. de Chimie Physique, 78(1) (1981) 85- 98

[3] - S.M. Haiji, M. Moussa et M. Waliou Ould Sellahi, Première Journée Nationale sur les Micelles, les microémulsions et les Tensioactifs, Settat, Mar oc, 11 Mars (1999)

[4] - E. Vikingstad, A. Skauge and H. Hailand, J. Colloid and Interf. Sci., 66(2) (1978) 240-6

[5] - T. Nakajima, T. Komatsu and T. Nakagawa, Bulletin Chem. Soc. Jap., 48(3) (1975) 383-7

[6] - M. S. Haiji, "Thèse de doctorat d'état ès sciences physiques", Univ. Paris Nord, (1987)

[7] - R. Coudert, II Thèse de doctorat d'état ès sciences physiques II, Univ. Paris Nord, (1984)

[8] - R. Umemura, D.G. Cameron and H.H. Mantch, J. Phys. Chem., 8 (1980) 2272-77

[9] - E. Edward and J. King, J. Phys.Chem., 84 (1974) 1217-25

[10] - U. Henricksson and L. Odberg, coll. Poly. Sci., 254 (1976) 35-8

[11] - S. Lindenbaum, J. Chem. thermodyn., 3 (1971) 625-9

[12]- M. Sakurai, T. Komatsu and T. Nakagawa, Bulletin Chem. Soc. Jap., 48(12) (1975) 3491-95

[13] - P.A. Leduc and J.E. Desnoyers, Can. J. Chem., 51 (1973) 2993-98

[14] - K. Watanabe, M. Ferrario and M. L. Klein, J. Phys. Chem., 92 (1989) 819-21

[15] - E. M. Woolley and T. E. Burchfield, J. Phys. Chem., 89 (1985) 714-22

[16] - J.B. Rosenholm, T. E. Burchfield, and I.G. Hapler, J. of Colloid and Interf. Sci., 78(1) (1980) $191-4$

[17] - J.E. Desnoyers, R. Delisi, G. Perron, Pure \& Apl. Chem., 52 (1980) $433-44$

[18] - J.T.G. Overbeek, Chemisch Weekblad Nederl., 54 (1958) 687-91

[19] - K.M. Kale, R. Zana, J. Colloid and Interf. Sci., 61(2) (1977) 313-22

[20] - P. Debye, Chem. Weekblad, 1948; J. Phys. and Colloid Chem., 53, (1949), 1; Ann., N.Y., Acad. Sci., 51 (1949) 575 
[21] - D. Stigter and J.T.G. Overbeek, Rec. Trav. Chem., 75 (1956) 1263; Proc. 2nd International Congess of Surface Activity / Gaz and Liquid / Interface. Butterworth, London, (1957) 311

[22] - E. W. Anacker and A. L. Underwood, J. Phys. Chem., 85 (1981) 2463-66

[23] - Landolt-Bornstein, New-Series IV/lb "Densities of binary aqueuse systems and heat capacities of liquid systems" Springer-verlag, Berlin-Heidelberg-New-york, (1977) 1

[24] - G. S. Kell, J. Chem. Eng. Data, 12 (1967) 66

[25] - O. Redlich and D. M. Meyer, Chem. Rev., 64 (1964) $221-7$

[26] - L. A. Dunn, Trans. Faraday Soc., 64 (1968) 2951

[27] - R. G. Bates, "Dermination of pH", Wiley, N. Y., (1964) 406

[28] - P.A. Leduc, Can. J. Chem., 51 (1973) 2993

[29] - E. M. Wolley and E. B. Thomas, J. Phys. Chem., 88 (1984) 2155-63

[30] - E. B. Thomas and E. M. Wolley, J. Phys. Chem., 88 (1984) 2149-55

[31] - R. F. Kamrath and E. I. Francess, Ind. Eng. Chem. Fundam., 22 (1983) 230

[32] - H. B. Clevens, J. Amer. Oil. Chem. Soc., 30 (1953) 74

[33] - M. Eigen and E. Wicke, Z. Elektro Chem., 55 (1951) 354

[34] - C. Damas, L Vanier, M Abarbi, A Duchene and R Coudert, J. Colloid and Interf. Sci., 198 (1998) 323-9

[35] - R. Coudert, M.S. Haiji and A Cao, J. of Colloid and Interf. Sci., 155 (1993) 173-182

[36] - K Mugero, Y Takasawa, N Kawahashi, Y. Tabata and M. Ueno, J. of Colloid and Interf. Sci., 83(1) (1981) 50

[37] - M. Sakurai, Bulletin Chem. Soc. Jap., 46(6) (1973) 1596-1602

[38] - J. E. Desnoyers et M. Arel, Can. J. Chem., 45 (1967) 359

[39] - F. Frank and H. T. Smith, J. Phys. Chem., 68 (1964) 3581

[40] - S. M. Haiii, M. B. Errahmani, R. Coudert, R. R. Durand, A. Cao and E. Taillandier, J. Phys. Chem., 93 (1989) 4818-24

[41] - C. Jolicoeur and G. Lacroix, Can. J. Chem., 54 (1976) 624

[42] - E. Vikingstad, J. of Colloid and Interf. Sci., 73(2) (1980) 500

[43] - 0. Redlich and L E Nielson, J. Amer. Chem. Soc., 64 (1942) 761-2

[44] - E. Vikingstad, A. Skauge and H. Hoiland, J. of Colloid and Interf. Sci., 66(2) (1978) 240-6

[45] - G. S. Harteley "Micellization, Solubilisation and Microemulsion", Edité par K. L. Mittal, Plenum Press, N. Y., (1977) 23

[46] - P. Ekwall, H. Eikrem and L. mandell, Acta. Chem. Scand., 17(1) (1963) 111-22

[47] - N. A. Campbell, E M Kartzmark et G. R. Lakshminarayanan, Can. J. Chem., 40 (1962) 831 
[48] - J. Umemura, H. H. Mantsch and D. G. Cameroun, J. of Colloid and Interf. Sci., 83(2) (1981) $558-68$

[49] - K. Hess, W. Philipoff and H. Kiessig, Kolloid J., 40 (1939) 88

[50] - Hayter and J. B. Penfold, J. Chem. Soc. Faraday Trans., I (1981)

[51] - N. A. Campbell et G. R. Lakshminarayanan, Can. J. Chem., 43 (1965) 1729. 